\title{
Arc statistics in cosmological models with dark energy
}

\author{
M. Bartelmann ${ }^{1}$, M. Meneghetti ${ }^{1,2}$, F. Perrotta ${ }^{3,4,5}$, C. Baccigalupi ${ }^{4,5}$, and L. Moscardini ${ }^{6}$ \\ 1 Max-Planck-Institut für Astrophysik, PO Box 1317, 85741 Garching, Germany \\ 2 Dipartimento di Astronomia, Università di Padova, vicolo dell'Osservatorio 2, 35122 Padova, Italy \\ 3 Osservatorio Astronomico di Padova, Vicolo dell'Osservatorio 5, 35122 Padova, Italy \\ ${ }^{4}$ Lawrence Berkeley National Laboratory, 1 Cyclotron Road, Berkeley, CA 94720, USA \\ 5 SISSA/ISAS, via Beirut 4, 34014 Trieste, Italy \\ ${ }^{6}$ Dipartimento di Astronomia, via Ranzani 1, 40127 Bologna, Italy
}

Received 2 October 2002 / Accepted 18 July 2003

\begin{abstract}
We investigate how the probability of the formation of giant arcs in galaxy clusters is expected to change in cosmological models dominated by dark energy with an equation of state $p=w \rho c^{2}$ compared to cosmological-constant or open models. To do so, we use a simple analytic model for arc cross sections based on the Navarro-Frenk-White density profile which we demonstrate reproduces essential features of numerically determined arc cross sections. Since analytic lens models are known to be inadequate for accurate absolute quantifications of arc probabilities, we use them only for studying changes relative to cosmological-constant models. Our main results are (1) the order of magnitude difference between the arc probabilities in low density, spatially flat and open CDM models found numerically is reproduced by our analytic model, and (2) dark-energy cosmologies with $w>-1$ increase the arc optical depth by at most a factor of two and are thus unlikely to reconcile arc statistics with spatially flat cosmological models with low matter density.
\end{abstract}

Key words. galaxies: clusters: general - cosmology: theory - cosmology: dark matter - cosmology: gravitational lensing

\section{Introduction}

The statistics of giant luminous arcs in the cores of galaxy clusters has long been recognised as a potentially powerful cosmological probe (e.g. Wu \& Mao 1996; Bartelmann et al. 1998). Arcs are formed by gravitational lensing from sources which happen to lie close to the caustic curves of a cluster lens, where magnification and distortion are particularly strong.

The cosmological power of arc statistics derives from at least two, maybe three principal reasons. First, for clusters to be efficient lenses, they have to be located approximately half-way between the sources, typically around redshift unity, and the observer. Depending mostly on the mean cosmic matter density, parameterised by $\Omega_{0}$, clusters form earlier or later in cosmic history if the matter density is low or high, respectively. In high-density model universes, the cluster population at the redshifts mostly relevant for lensing, $z \sim 0.3-0.4$ is substantially less rich than in low-density universes, reducing the number of available efficient lenses dramatically (e.g. Richstone et al. 1992; Bartelmann et al. 1993; Lacey \& Cole 1993, 1994).

The second principal reason is that strong lensing is a highly nonlinear phenomenon in the sense that it requires the lensing mass distribution to be supercritical for strong lensing, which means that a suitable combination of surface mass

Send offprint requests to: M. Bartelmann, e-mail: msb@mpa-garching.mpg.de density and gravitational tidal field needs to be large enough, and that, once a lens is supercritical, even small changes in both can change significantly the length of the caustic curves, and thus the lens' ability for strong lensing.

Different cosmological models predict the mass distribution in clusters to be more or less concentrated. Numerical simulations consistently show that, the earlier a dark-matter halo forms, the more concentrated it is because it appears to keep a record of the mean cosmic density at the time when it formed (Navarro et al. 1996, 1997). Structure forms later in spatially flat than in open, low density cosmological models, thus halos in models with cosmological constant are generally less concentrated than halos in open models.

A possible third reason is that the gravitational tidal field at the location of the lens plays a very important role (Bartelmann et al. 1995; Meneghetti et al. 2001). It is strong if lenses are highly asymmetric, as clusters frequently are, and if the surrounding matter distribution is highly irregular. It is possible that, if cosmic structure forms later, cluster mass distributions are less relaxed and thus more asymmetric, and that also the degree of irregularity in their neighbourhood is different than if structures formed earlier. On the other hand, clusters forming earlier are built from subhalos which tend to be more concentrated and thus more strongly gravitationally bound, hence substructures could then persist within clusters for a longer time and contribute to the asymmetry. 
Unfortunately, the combination of these effects renders analytic models for arc statistics entirely inadequate for accurate quantitative predictions of arc probabilities (Meneghetti et al. 2003). The effects of cosmology on cluster compactness and asymmetry, and on the tidal field of the matter surrounding the clusters, cannot be captured by reasonably simple analytic lens models. Numerical simulations of arc statistics, using clusters formed in sufficiently large $N$-body simulations as lenses, led to the surprising result that the expected number of giant luminous arcs on the sky differs by orders of magnitude between different cosmological models. While a model with critical matter density and no cosmological constant fell below the observed number of arcs, extrapolated to the full sky, by two orders of magnitude, a $\Lambda$ CDM model with $\Omega_{0}=0.3$ failed by one order of magnitude, and only a low-density open model with $\Omega_{0}=0.3$ produced approximately the right number of arcs (Bartelmann et al. 1998).

The statistics of quasars multiply imaged by galaxies has often been used for constraining cosmological parameters. The basic argument is that the number of lenses and their redshifts should increase as $\Omega_{\Lambda}$ increases, which typically yields upper limits on $\Omega_{\Lambda} \lesssim 0.6-0.7$ (Kochanek 1996; Falco et al. 1998; Quast \& Helbig 1999), although discrepant results have also been found (Chiba \& Yoshii 1999; Helbig 1999; Keeton 2002). We emphasise that the sensitivity of cluster lensing to $\Omega_{\Lambda}$ is of a different nature. Since clusters form much later in cosmic history than galaxies, the volume effect is negligible, but $\Omega_{\Lambda}$ changes the dynamics of cluster formation and thus their core structure, to which strong lensing is highly sensitive.

These numerical results of Bartelmann et al. (1998) were tested by Cooray (1999) and Kaufmann \& Straumann (2000) using analytic models based on singular isothermal spheres. They could confirm the sensitivity of arc statistics to $\Omega_{0}$, but found only a very weak dependence on $\Omega_{\Lambda}$, in contrast to the numerical results. The isothermal sphere has two disadvantages with respect to arc statistics. First, arc cross sections are very sensitive to asymmetries in the cluster mass distribution, thus axially symmetric models lack a property which is crucially important for arc statistics. Second, numerical simulations show that the central density concentration of clusters depends on cosmology, and this potentially important feature is not reproduced by the scale-free isothermal models either. We shall construct in this paper an analytic model which qualitatively reproduces the earlier numerical results.

Bartelmann et al. (1998) used two completely different types of $\mathrm{N}$-body codes for simulating galaxy clusters. Numerous subsequent tests of the results showed that the arc numbers derived could be off by factors of perhaps 1.5 to 2 , but that there was no way how order-of-magnitude differences could be bridged (Meneghetti et al. 2000; Flores et al. 2000). The problem became substantially more acute when measurements of the cosmic microwave background (CMB), combined with observations of supernovae of type Ia and large-scale galaxy surveys, left very little room for model universes which are not spatially flat and have density parameters much different from $\Omega_{0} \approx 0.3$ (e.g. Riess et al. 1998; Perlmutter et al. 1999; Lee et al. 2001; Abroe et al. 2002; Efstathiou et al. 2002; Netterfield et al. 2002; Wang et al. 2002;
Halverson et al. 2002). Obviously, there is an interesting discrepancy between the statistics of arcs seen on the sky, and the probability for arcs produced in cosmological models which are convincingly required by various other observations. While observations consistently indicate a high probability for arc formation in clusters (Le Fèvre et al. 1994; Gioia \& Luppino 1994; Luppino et al. 1999; Zaritsky \& Gonzalez 2003; Gladders et al. 2003), we should point out that the discrepancy between theory and observations is so far only based on a single set of simulated clusters.

A spatially flat universe with low matter density and a cosmological constant is extremely difficult to justify theoretically. The vacuum energy density provided by the cosmological constant is tens of orders of magnitudes below any natural scale which is conceivable in particle physics (see Carroll 2001 for a review on the cosmological constant problem). This difficulty motivated the introduction of a more general concept for a vacuum energy cosmological component, now widely known as dark energy. The theoretical and observational aspects of the dark energy are one of the most important issues in modern cosmology (see Peebles \& Ratra 2002 for a review). In general, the most important difference of a dark energy component compared to a cosmological constant is that its equation of state, $w$, can be different from -1, generally implying a time variation. It should be noted that recent analyses of CMB data seem to favour a value of $w$ very close to -1 , albeit these results suffer to some degree from parameter degeneracies and are typically obtained under restrictive assumptions (Douspis et al. 2003; Melchiorri et al. 2003; Spergel et al. 2003).

Recently, Bartelmann et al. (2002) argued that dark matter halos in simple dark energy models should be more concentrated than in cosmological constant models with the same dark energy density today. The main reason is that halos form earlier in dark energy models, allowing them to be more compact. Several different recipes for describing halo concentrations found in numerical simulations as a function of their formation time lead to consistent results. The halo concentration increases noticeably in the interval $-1 \leq w \leq-0.6$. For higher values of $w$, which are too high for the cosmic acceleration to agree with recent data (Riess et al. 1998; Perlmutter et al. 1999), there is a strongly opposing effect related to the amplitude of fluctuations in the CMB: The high level of the Integrated Sachs-Wolfe (ISW) effect on the large scale CMB anisotropies leads to a sharp decrease in the normalisation of the dark matter power spectrum (Bartelmann et al. 2002). Within a cosmologically interesting range for the equations of state of the dark energy, the balance between the ISW effect and the earlier formation of halos is quite delicate, but halos can typically be expected to be more concentrated.

The ability of a galaxy cluster to produce giant arcs depends sensitively on the concentration of its mass profile because of the nonlinearity of the strong lensing effect. We therefore wish to investigate how the probability for arc formation changes in dark energy models, compared to cosmological-constant or open models. For doing so, we use a simple, analytic description for the arc cross section of a cluster of given mass, which we demonstrate to possess the relevant features of the fully numerical results. Although it has been shown that analytic 
models are inadequate for quantitatively reliable arc statistics, we are here interested only in the relative change of the arcformation probability caused by changes in the cosmological model.

Section 2 of the paper introduces the cosmological background model. Section 3 describes our simple analytic model for the arc cross section of a galaxy cluster. We then use this model in Sect. 4 for calculating arc probabilities, and summarise our conclusions in Sect. 5.

\section{Cosmological model}

Dark energy is characterised by a negative pressure, $p=w \rho c^{2}$, where $\rho c^{2}$ is the mean energy density of the universe and the equation of state $w$ assumes negative values in order to produce cosmic acceleration according to the data from type Ia supernovae (Riess et al. 1998; Perlmutter et al. 1999). Theoretical models of dark energy, such as Quintessence scalar fields, generally predict a time variation of the equation of state, as well as the presence of dark energy fluctuations on super-horizon cosmological scales (see e.g. Peebles \& Ratra 2002 and references therein). In this work, we concentrate on the very basic aspect of dark energy, by neglecting the spatial inhomogeneities and assuming $w$ to be a constant. In this case, the adiabatic equation requires the equivalent matter density $\rho_{\mathrm{Q}}$ of the dark energy to change with the cosmological scale factor $a$ as

$\rho_{\mathrm{Q}}=\rho_{\mathrm{Q}, 0} a^{-3(1+w)}$,

starting from the density $\rho_{\mathrm{Q}, 0}$ today. Obviously, cosmological constant models are retained setting $w=-1$. Replacing the conventional cosmological-constant term by a dark-energy term, Friedmann's equation reads

$H^{2}(a)=H_{0}^{2}\left[\Omega_{0} a^{-3}+\Omega_{\mathrm{Q}} a^{-3(1+w)}\right]$,

assuming with support from recent measurements of anisotropies in the cosmic microwave background that the curvature term is negligible. Here, $H(a)$ is the Hubble parameter as a function of $a, H_{0}$ is the Hubble constant, and $\Omega_{0}$ is the density parameter for non-relativistic matter.

The main consequences for the structure formation process of this modified term in Friedmann's equation have been detailed in an earlier paper (Bartelmann et al. 2002), so we summarise them only briefly here. Starting from a low-density cosmological constant model, keeping $\Omega_{0}$ fixed and increasing $w$, the cosmic volume per unit redshift shrinks, and the linear growth factor for cosmic structures starts rising earlier, hence structures start forming earlier if the cosmological constant is replaced by a dark energy component. Additional effects are that the parameters characterising halo formation, i.e. the linear overdensity $\delta_{\mathrm{c}}$ and the virial overdensity $\Delta_{\mathrm{v}}$, are changed (cf. Wang \& Steinhardt 1998; Mainini et al. 2003).

As we anticipated in the introduction, a further important consequence of dark energy models is that the gravitational potential of the density fluctuations changes more rapidly with time, leading to an increased integrated Sachs-Wolfe effect on the CMB fluctuations at large angular scales. Given the observed level of CMB power on large angular scales ${ }^{1}$, a decreasing fraction of the observed anisotropies can thus be attributed to the primordial CMB fluctuations, hence the normalisation of the power spectrum has to be reduced as $w$ is increased in order to be compliant with the COBE-DMR data. The main result is that the power-spectrum normalisation $\sigma_{8}$ is decreasing gently as $w$ is increased from -1 to $\sim-0.6$, and then turns to drop more rapidly as the ISW effect intensifies. Here and below, we adopt the CDM power spectrum with the Harrison-Zel'dovich power-law index $n=1$ and the transfer function given by Bardeen et al. (1986). Throughout, we use $\Omega_{0}=0.3$ and $\Omega_{\mathrm{Q}}=0.7$. It should be noted here that our results will be sensitive to the exact value of $n$ as well as other cosmological parameters.

\section{Arc cross sections}

\subsection{Halo model}

We will assume in the following that the average radial density profile of galaxy clusters can be described by the profile found in numerical simulations by Navarro et al. (1996, hereafter NFW),

$\rho(r)=\frac{\rho_{\mathrm{s}}}{r / r_{\mathrm{s}}\left(1+r / r_{\mathrm{s}}\right)^{2}}$,

where $\rho_{\mathrm{s}}$ is a density scale, and $r_{\mathrm{s}}$ a scale radius. The ratio between $r_{\mathrm{s}}$ and the radius $r_{200}$ enclosing a mean halo density of 200 times the critical density is called concentration, $c=r_{\mathrm{s}} / r_{200}$. The two parameters, $\rho_{\mathrm{s}}$ and $r_{\mathrm{s}}$, are not independent. Numerical simulations showed that the concentration parameter $c$ depends on the halo mass, which is thus the only free parameter.

Numerically simulated halos tend to be the more concentrated the earlier they form. Their central density apparently reflects the mean cosmic density at the time of their formation. Since halos of higher mass are formed later in hierarchical models than halos of lower mass, the concentration is decreasing with halo mass. Based on these findings, several algorithms were designed for computing halo concentrations from halo masses.

The algorithm by Navarro et al. (1997) first assigns to a halo of mass $M$ a collapse redshift $z_{\text {coll }}$ defined as the redshift at which half of the final halo mass is contained in progenitors more massive than a fraction $f_{\mathrm{NFW}}$ of the final mass. Then, the density scale of the halo is assumed to be some factor $C$ times the mean cosmic density at the collapse redshift. They recommend setting $f_{\mathrm{NFW}}=0.01$ and $C=3 \times 10^{3}$ because their numerically determined halo concentrations were well fit assuming these values.

Bullock et al. (2001) suggested a somewhat simpler algorithm. Haloes are assigned a collapse redshift defined such that

\footnotetext{
${ }^{1}$ The only existing data are from the Differential Microwave Radiometer (DMR) on board the COsmic Background Explorer satellite (COBE, see Smoot 1999 and references therein); more data will be available in the near future from the Microwave Anisotropy Probe (MAP, map.gsfc.nasa.gov) and the Planck (astro.estec.esa. $\mathrm{nl} / \mathrm{SA}$-general/Projects/Planck) satellites.
} 


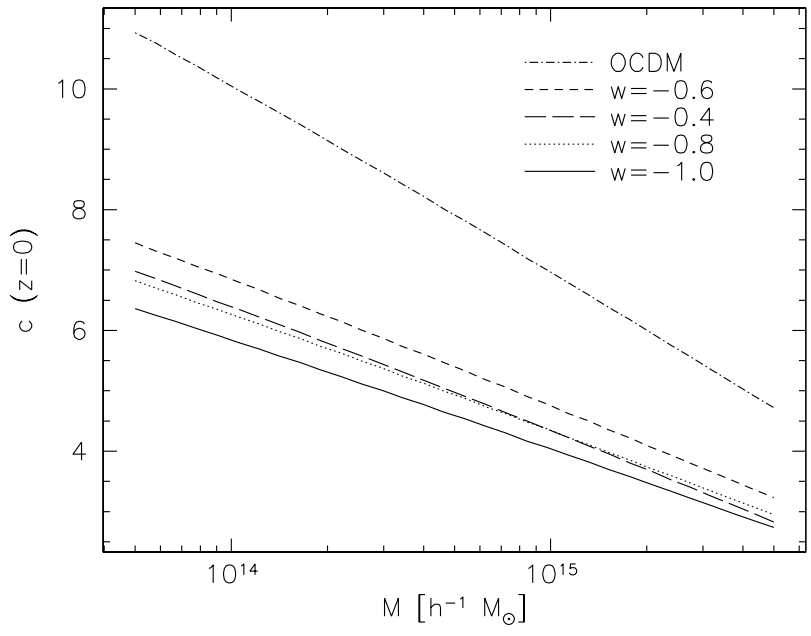

Fig. 1. Halo concentrations according to Eke et al. (2001) are shown as functions of halo mass for four spatially flat cosmological models with different choices for the dark energy parameter $w$ and for the OCDM model, as indicated. As $w$ increases, halos become more concentrated until $w \approx-0.6$. If $w$ increases further, halo concentrations drop because then the amplitude $\sigma_{8}$ of the power spectrum has to decrease rapidly in order to remain consistent with the COBE-DMR data, as the integrated Sachs-Wolfe effect becomes larger (cf. Bartelmann et al. 2002).

the non-linear mass scale at that redshift is a fraction $f_{\mathrm{B}}$ of the final halo mass. The halo concentration is then assumed to be a factor $K$ times the ratio of the scale factors at the redshift when the halo is identified and at the collapse redshift. Comparing with numerical simulations, they found $f_{\mathrm{B}}=0.01$ and $K=4$. This algorithm reflects the change of halo concentrations with redshift more accurately than the approach by Navarro et al. (1997) predicts.

A third algorithm was suggested by Eke et al. (2001). They assigned the collapse redshift to a halo of mass $M$ by requiring that the suitably defined amplitude of the linearly evolving power spectrum at the mass scale $M$ equals a constant $C_{\mathrm{ENS}}^{-1}$. Numerical results are well represented setting $C_{\text {ENS }}=28$.

We will adopt the latter method for this paper because the concentrations computed from the algorithm by Navarro et al. drop too slowly with redshift compared to numerical simulations, and the algorithm by Bullock et al. has problems for high halo masses because of the requirement that a fixed fraction of the final halo mass should equal the nonlinear mass, which may never be reached if the halo mass is high. Concentrations as a function of halo mass for four different choices of the dark energy parameter $w$ are shown in Fig. 1 .

\subsection{Strong lensing by NFW haloes}

The arc cross section of a galaxy cluster is the area in the source plane where a source has to lie for being imaged as an arc with specified properties, e.g. exceeding a threshold length-to-width ratio. A typical cluster lens has two critical curves, defined as curves in the lens plane along which the Jacobian matrix of the lens mapping is singular, and the image magnification is formally infinite (cf. Fig. 2). The critical curves are the images of

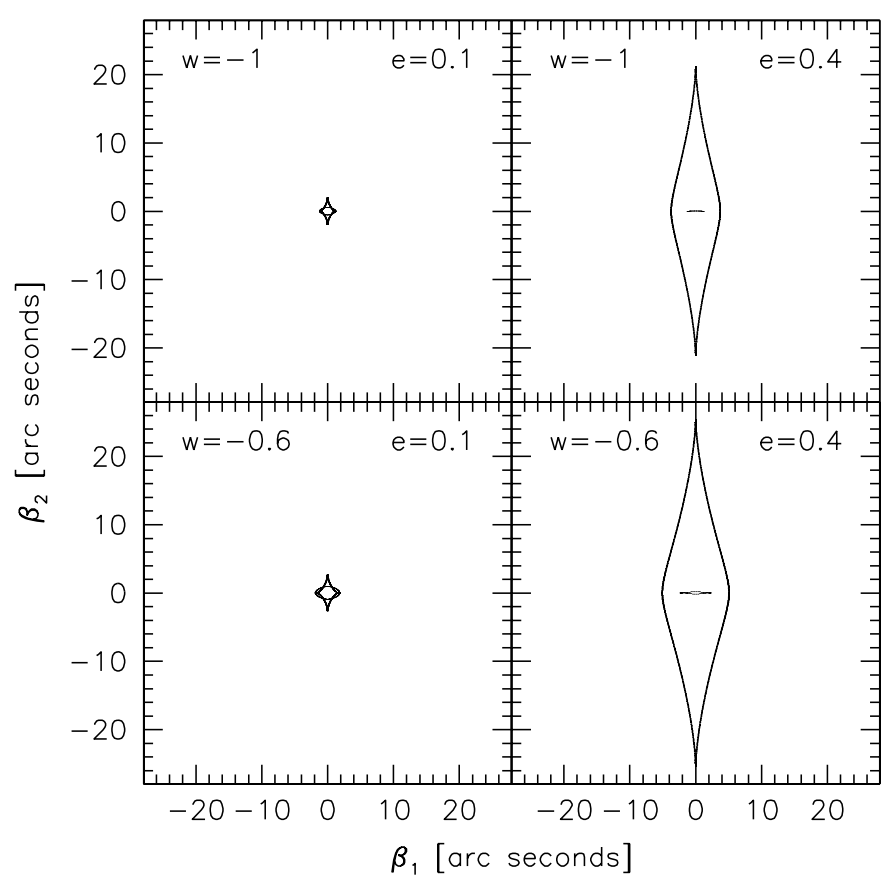

Fig. 2. Tangential and radial caustics for NFW lens models with elliptically distorted lensing potential. A halo of mass $10^{15} h^{-1} M_{\odot}$ at redshift $z=0.3$ is assumed here, and the sources are all placed at $z_{\mathrm{s}}=1$. The cosmological model is spatially flat with $\Omega_{0}=0.3$ and normalised to the COBE-DMR data. The upper panels show the caustics for a dark energy parameter of $w=-1$ and for two ellipticities, $e=0.1$ and $e=0.4$. The lower two panels show the caustics for $w=-0.6$. The figure illustrates the high sensitivity of strong lensing to halo ellipticity and concentration.

the caustic curves, thus sources close to a caustic are imaged as highly magnified and distorted images. The two critical curves and their corresponding caustic are called tangential and radial, because of the dominant orientation of the image distortion relative to the centre of the lens. Seeking to quantify large-arc cross sections, we are thus looking for an appropriately defined area covering the tangential caustic of a cluster.

It is well known that arc cross sections depend strongly on the exact shape of the cluster mass distribution, and on the gravitational tidal field exerted by density fluctuations in its neighbourhood (Bartelmann et al. 1995). Thus, the only reliable method for exactly quantifying arc cross sections has to use numerically simulated cluster models without referring to any symmetry assumptions. For a study like ours, however, we only need to describe how arc cross sections are expected to change relative to a fiducial model when certain cosmological parameters are modified.

Gravitational lensing by an NFW halo can be described by its lensing potential (e.g. Bartelmann 1996; Meneghetti et al. 2003),

$\psi(x)=4 \kappa_{\mathrm{s}}\left[\frac{1}{2} \ln ^{2} \frac{x}{2}-2 \operatorname{arctanh}^{2} \sqrt{\frac{1-x}{1+x}}\right]$,

which is related to the lensing convergence through the Poisson equation

$\kappa(x)=\frac{1}{2} \nabla^{2} \psi(x)$ 
The factor $\kappa_{\mathrm{S}}$ in Eq. (4) is defined by

$\kappa_{\mathrm{s}}=\frac{\rho_{\mathrm{s}} r_{\mathrm{s}}}{\Sigma_{\mathrm{cr}}}$

where $\Sigma_{\text {cr }}$ is the critical surface mass density for lensing. The dimensionless radius $x=r / r_{\mathrm{s}}$ can conveniently be replaced by the angular radius $\theta=r / D_{\mathrm{d}}=x r_{\mathrm{s}} / D_{\mathrm{d}}$, where $D_{\mathrm{d}}$ is the angular diameter distance from the observer to the lens. The gravitational tidal field, or shear, of the lens is the two-component quantity

$\gamma_{1}=\frac{1}{2}\left(\psi_{11}-\psi_{22}\right), \quad \gamma_{2}=\psi_{12}$,

where the subscripts abbreviate partial derivatives with respect to the angular coordinates $\left(\theta_{1}, \theta_{2}\right)$ on the sky. The deflection angle is the gradient of the lensing potential,

$\alpha(\theta)=\nabla \psi(\theta)$.

The Jacobian matrix of the lens mapping has the components

$\mathcal{A}_{i j}=\delta_{i j}-\psi_{i j}$

Its eigenvalues are $\lambda_{ \pm}=(1-\kappa) \pm \gamma$, where $\gamma=\left(\gamma_{1}^{2}+\gamma_{2}^{2}\right)^{1 / 2}$ is the amplitude of the shear. The tangential critical curve is determined by the condition $\lambda_{-}=(1-\kappa)-\gamma=0$.

We now distort the axially symmetric NFW lens such that the iso-potential lines become ellipses,

$\psi(\theta) \rightarrow \psi(\vartheta), \quad \vartheta=\left[\frac{\theta_{1}^{2}}{1-e}+\theta_{2}^{2}(1-e)\right]^{1 / 2}$

As noted by Kassiola \& Kovner (1993), an elliptical potential can lead to dumbbell-shaped mass distributions with locally negative mass density. As real clusters are irregular, dumbbellshaped mass distributions are acceptable. For the NFW profile with elliptical isopotential contours, the mass density does indeed become mildly negative, but only well outside the core where strong lensing occurs. For $e=0.4$, the minimum $\kappa$ is $\sim-0.01$ times the convergence in the core (see also Golse \& Kneib 2002). We thus use the elliptical lensing potential (10) for computational simplicity.

The Jacobian matrix and its eigenvalues can be computed from (10) using the relations introduced before. Generally, the zeroes of the tangential eigenvalue $\lambda_{-}$have to be determined numerically. On the coordinate axes, they are given by

$\vartheta=\left\{\begin{array}{ll}(1-e) \alpha & \left(\theta_{2}=0\right) \\ \frac{\alpha}{1-e} & \left(\theta_{1}=0\right)\end{array}\right.$.

The corresponding caustic points, i.e. the cusps of the diamondshaped caustic on coordinate axes, can then be found using the lens equation,

$\beta=\theta-\alpha(\theta)$.

We thus know the four intersection points of the tangential caustic curve with the coordinate axes. We defined them to lie at $\left(\theta_{1}, \theta_{2}\right)=(0, \pm a)$ and $\left(\theta_{1}, \theta_{2}\right)=( \pm b, 0)$. Since the major axis of the iso-potential ellipses points along the $\theta_{2}$ axis, the cusps on the $\theta_{2}$ axis are further away from the lens centre than the cusps on the $\theta_{1}$ axis, hence $a>b$. Figure 2 illustrates the caustic curves for elliptical NFW lens models with two different ellipticities in two spatially flat cosmological models with different values for $w$.

A simple assumption for the large-arc cross section $\sigma$ of the elliptically distorted NFW lens holds that it is proportional to the area enclosed by the critical curve, hence $\sigma \propto a b$, with a proportionality constant depending on the exact shape of the caustic curve, and thus on the ellipticity of the lens model. Since we do not require an absolute calibration of the arc cross sections, we adopt $\sigma=a b$. Earlier work (Meneghetti et al. 2000; Flores et al. 2000) has shown that individual galaxies have a negligible effect on arc cross sections, which further supports the assumption that they are determined by the overall extent of the caustic curves.

We will later have to integrate over the cluster population, conveniently parameterised by the virial mass and described by the mass function. Thus, we have to verify whether our approximate description of the arc cross section scales with cluster mass in the same way as numerically determined arc cross sections of the same cluster models, given identical ellipticity parameters $e$. We therefore set up deflection-angle maps starting from the elliptically distorted lensing potential $\psi(\vartheta)$ and used them for imaging randomly distributed, intrinsically elliptical sources by tracing rays passing the image plane on a rectangular grid. The sources are placed on adaptively refined grids whose resolution is progressively increased in the vicinity of caustic curves. The images are automatically classified according to their length, width, length-to-width ratio and several other parameters, and the cross section is determined by counting the number of images exceeding a threshold lengthto-width ratio. More detail on this method can be found in (Bartelmann \& Weiss 1994; Meneghetti et al. 2000). Results are shown in Fig. 3.

The essential feature of the numerically determined arc cross sections is that they increase approximately quadratically with the lens mass. Since the Einstein radius of an extended lens typically scales linearly with the lens mass, this indicates that the arc cross section scales approximately like the square of the Einstein radius. Our simple estimate for the arc cross section is defined to reproduce this property.

We also check whether numerically determined cross sections scale with the dark energy parameter $w$ in a similar way as our simple cross-section estimate does. Figure 4 shows the example of an elliptically distorted NFW lens of mass $M=$ $7.5 \times 10^{14} h^{-1} M_{\odot}$. The curves plotted there show qualitatively the same behaviour. As $w$ increases above -1 , the arc cross section increases by a factor of $\sim 1.9$ until $w \approx-0.6$, and then drops as the normalisation constraint requires to significantly reduce $\sigma_{8}$. Both curves are arbitrarily normalised to unity at their peaks. The differences between the numerically determined cross sections and our simple estimate are unlikely to be significant because the numerical method uses the images of a finite number of discrete sources for determining the cross sections, which causes noise in the results. In any case, the two essential properties of the change in the cross sections with $w$ are well reproduced, namely the amplitude of the relative increase and the location of the peak. 


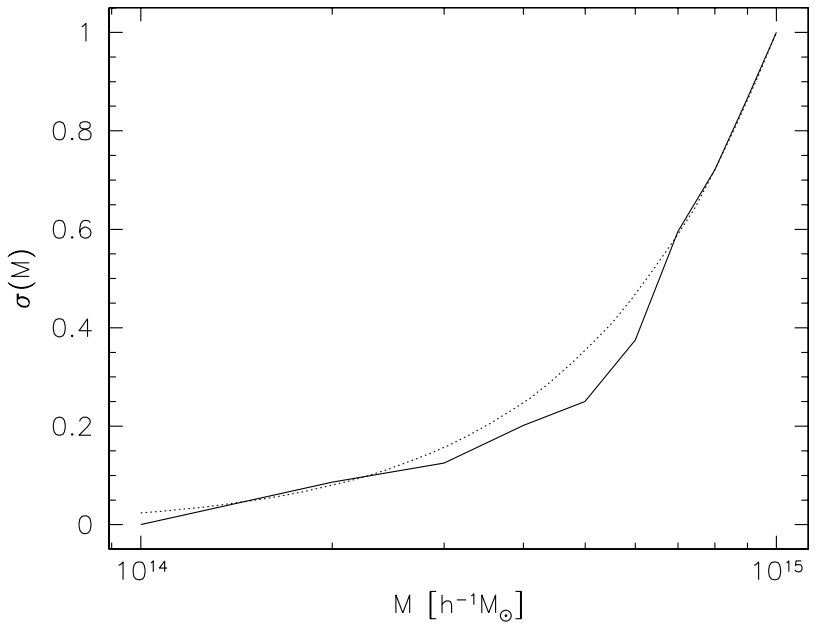

Fig. 3. Comparison between the numerically determined arc cross sections of elliptically distorted NFW lenses as a function of their virial mass $M$ (solid curve), and the simple estimate for the arc cross sections which is proportional to the area covered by the tangential caustic curve (dotted curve). Both curves are arbitrarily normalised to unity at the high-mass end. The lenses are placed at redshift $z=0.3$, the sources at redshift $z_{\mathrm{s}}=1.0$, the ellipticity of the NFW potential is $e=0.4$. The curves were computed for a dark energy cosmological model with $w=-0.6$. Evidently, the simple estimate for the cross sections correctly reproduces the scaling of the cross sections with cluster mass. The numerically determined curve is not smooth because the cross sections are computed from finite numbers of simulated arcs and thus subject to random fluctuations.

Finally, we set the cross section to zero if the major axis of the critical curve falls below some threshold $\theta_{\min }$. The idea behind this is that if the critical curves become too small, the images near the critical curves can hardly be called giant arcs. Suppose typical sources have diameters on the order of an arc second, and the lens should be able to produce arcs with a length-to-width ratio around ten. Then, ignoring the source magnification in the radial direction, the tangential critical curve needs to have a radius of approximately $10^{\prime \prime}$ for this to happen. We thus set $\theta_{\min }=10^{\prime \prime}$ unless stated otherwise, and show the effect of changing $\theta_{\min }$ to $5^{\prime \prime}$ below.

We conclude from this section that the scaling of our simple estimate for arc cross sections with lens mass and with the dark energy parameter $w$ well reproduces what is expected from numerical treatments of the same lens models, i.e. NFW lenses with elliptically distorted lensing potential. We emphasise again that the absolute value of the cross sections are unimportant for our present purposes, as we are aiming at studying the change in the arc-formation probability in various cosmological models relative to the $\Lambda \mathrm{CDM}$ model.

\section{Arc probabilities}

We can now proceed to compute the probability for arc formation by a population of clusters. Given a mass function $\mathrm{d} n(M, z) / \mathrm{d} M$, we can write the so-called optical depth $\tau\left(z_{\mathrm{s}}\right)$ as

$\tau\left(z_{\mathrm{s}}\right)=\int_{0}^{z_{\mathrm{s}}} \mathrm{d} z(1+z)^{3}\left|\frac{\mathrm{d} V}{\mathrm{~d} z}\right| \int_{M_{\min }}^{\infty} \mathrm{d} M \frac{\mathrm{d} n}{\mathrm{~d} M} \sigma(M, z)$,

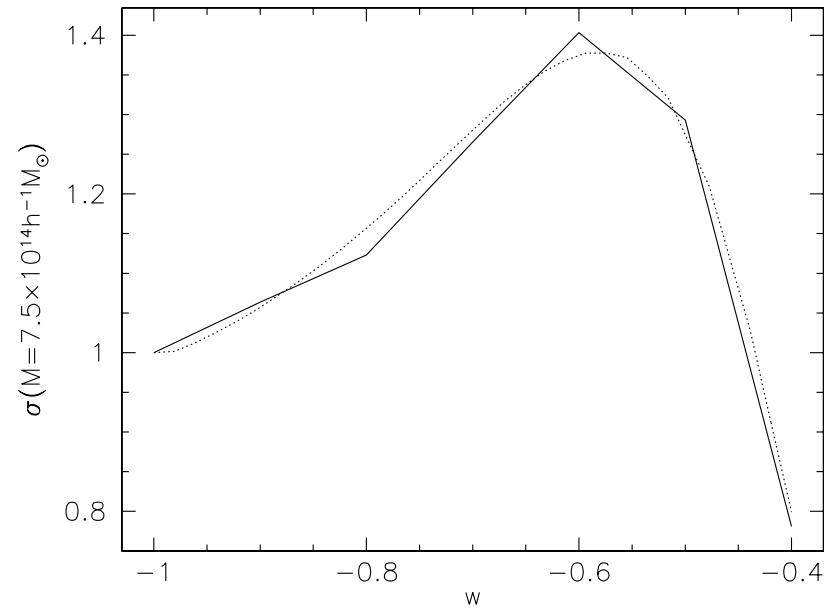

Fig. 4. Comparison between cross sections similar to Fig. 3, but for a fixed halo mass of $M=7.5 \times 10^{14} h^{-1} M_{\odot}$ and varying dark energy parameter $w$. The solid curve showing the numerically determined cross sections closely follows the dotted curve, which represents the simple cross-section estimate introduced here. The curves are arbitrarily normalised to unity at their starting point, i.e. at $w=-1$. Increasing $w$ from -1 to -0.6 increases the cross sections by a factor of $\sim 1.9$. As in Fig. 3, lens and source redshifts are set to 0.3 and 1, respectively, and the ellipticity of the NFW lensing potential is set to $e=0.2$.

where $z_{\mathrm{s}}$ is the source redshift, $V$ is the cosmic volume, and the factor $(1+z)^{3}$ accounts for the fact that the mass function is defined per comoving volume. The lower mass limit $M_{\text {min }}$ is determined by the mass required to produce critical curves whose major axis exceeds the threshold $\theta_{\min }$ introduced in the preceding section, thus it depends on the lens redshift $z$.

For the mass function $\mathrm{d} n / \mathrm{d} M$, we choose the modification by Sheth \& Tormen (1999) of the Press \& Schechter (1974) mass function. It well reproduces the halo mass functions found in numerical simulations. We take into account that our definition of mass differs slightly from Sheth \& Tormen's in that we use the mass enclosed by a sphere in which the mean density is 200 times the critical rather than the mean density. The mass function depends on cosmology through the normalisation of the power spectrum and the linear overdensity parameter derived from the spherical collapse model.

We note an important difference to strong lensing by galaxies. While the population of galaxy lenses is well described as isothermal spheres with number counts derived from observations (e.g. Lehár et al. 2000; Keeton 2002), the lack of wide-separation lenses argues against isothermal density profiles in cluster-scale lenses (Flores \& Primack 1994; Porciani \& Madau 2000). Baryonic physics changes the central density profiles of galaxy-scale halos, but is inefficient on cluster scales (e.g. Kochanek \& White 2001). Cluster mass functions derived from X-ray observations are found to agree well with theoretical predictions based on Press-Schechter type models (e.g. Reiprich \& Böhringer 2002). Thus modelling the cluster population with NFW density profiles and the Sheth-Tormen mass functions is well justified.

We show in Fig. 5 the redshift integrand of Eq. (13), i.e. the cosmic volume times the integral over mass of the mass function times the arc cross section. The figure thus illustrates the 


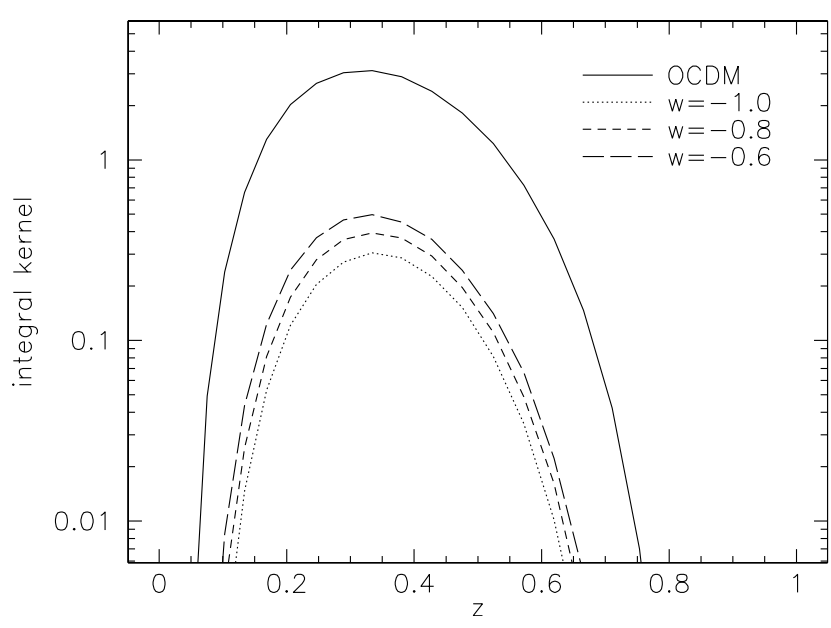

Fig. 5. The redshift integrand of Eq. (13) is plotted as a function of $z$ for four different cosmological models, a $\Lambda$ CDM model with $\Omega_{0}=0.3$, and open CDM model with $\Omega_{0}=0.3$ and no cosmological constant, and two spatially-flat dark energy models with $\Omega_{0}=0.3$ and $w=-0.8$ and $w=-0.6$. The spatially-flat models are normalised to the COBE-DMR data, and the open CDM model has the same $\sigma_{8}$ as the cosmological-constant model for easier comparison. The curves show that our simple analytic model succeeds in reproducing the order-of-magnitude difference between the open and the cosmological-constant model found in numerical simulations, and that spatially flat dark energy models cannot bridge the gap between these two models. The ellipticity of the NFW lensing potential was set to $e=0.3$ here

total arc cross section contributed by the cluster population at redshift $z$. For simplicity, sources are assumed to be at a single redshift of $z_{\mathrm{s}}=1$ here. The four curves in Fig. 5 are for the $\Lambda \mathrm{CDM}$ model with $\Omega_{0}=0.3$, the open CDM model with the same $\Omega_{0}$ but with $\Omega_{\Lambda}=0$, and for dark energy models with the same $\Omega_{0}$ but $\Omega_{\mathrm{Q}}=0.7$, and $w=-0.6$ or $w=-0.8$. Again, the curves are arbitrarily normalised such that the optical depth $\tau$ of the OCDM model is unity. The value of $\sigma_{8}=0.88$ of the $\Lambda$ CDM model was also set for the OCDM model for easier comparison. The COBE-DMR data would require $\sigma_{8} \sim 0.4$ for the OCDM model, which is way below the value required for reproducing the observed number density of massive clusters. We thus have to choose $\sigma_{8}$ for the OCDM model in conflict with the COBE-DMR data in order to produce comparable results on arc statistics.

Figure 5 shows two important results. First, the simple model for arc cross sections introduced here is capable of reproducing the order-of-magnitude difference in the total arc cross section between the $\Lambda \mathrm{CDM}$ and the OCDM models that had been found earlier in numerical simulations, and could not be reproduced by analytic models based on singular isothermal cluster mass distributions. Second, although the dark energy models have a somewhat higher total arc cross section than the $\Lambda \mathrm{CDM}$ model, they are still by a factor of $\sim 6$ below the arc cross section for the OCDM model. According to our analytic estimates, dark energy models are thus unable to reconcile spatially flat cosmological models with low matter density with the high abundance of large arcs, which seems to be similarly

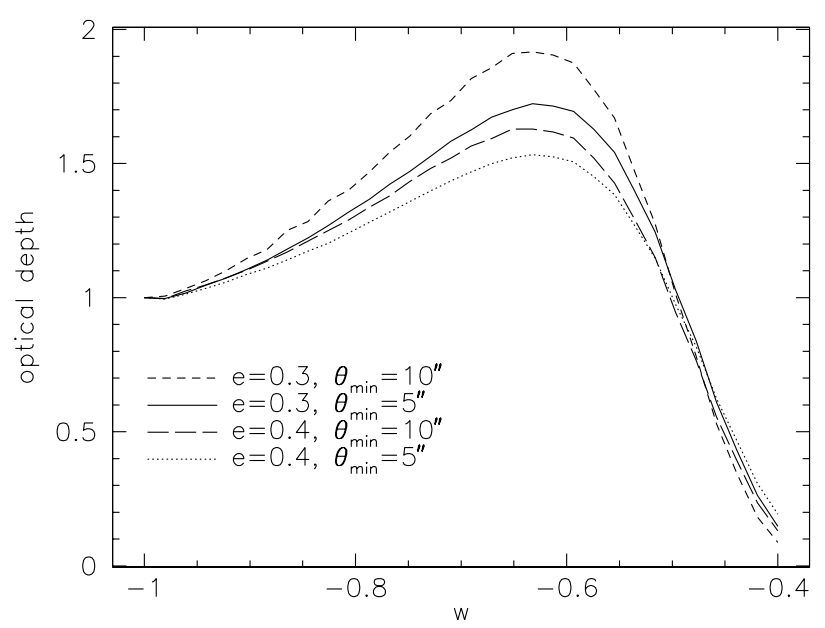

Fig. 6. Optical depth $\tau\left(z_{\mathrm{s}}=1\right)$ defined by Eq. (13) for spatially-flat dark energy models as functions of $w$. All curves are arbitrarily normalised to their values for $w=-1$, i.e. they show the change in optical depth with $w$ relative to the cosmological-constant model. All curves were obtained for $\Omega_{0}=0.3$ and COBE-normalised CDM power spectra. Results are shown for two different choices each for the ellipticity $e$ and the cutoff angle $\theta_{\min }$, as indicated. The curves show that clusters in dark energy models can be $\sim 50 \%$ up to $\sim 100 \%$ more efficient in forming arcs than in cosmological-constant models, depending in detail on the exact choices for the ellipticity and the cutoff angle.

impossible with cosmological constant models given the earlier numerical results.

Figure 6 shows the optical depth $\tau$ as defined in Eq. (13) for four different choices of the free parameters we have introduced, namely the ellipticity $e$ of the lenses and the cutoff radius $\theta_{\min }$. All curves have in common that the arc optical depth increases noticeably as $w$ increases from -1 to $\sim-0.6$, and drops rapidly as $w$ is increased further. The curves are intended to show the relative increase in $\tau$ compared to the $\Lambda \mathrm{CDM}$ model and are thus normalised to unity at $w=-1$. The peak amplitudes range from 1.5 to 2 . The largest increase is achieved for $\theta_{\min }=10^{\prime \prime}$ and $e=0.3$, the smallest for $\theta_{\min }=5^{\prime \prime}$ and $e=0.4$.

Several effects act jointly here. First, we saw in Fig. 4 that the arc cross section of (one example for) an individual halo increases by a factor of $\lesssim 2$ as $w$ is increased from -1 to -0.6 . An additional effect is that, as the halo concentration increases, halos of lower mass become capable of strong lensing. Since the mass function of galaxy clusters is steep, a small extension of the mass range towards lower masses can markedly increase the number of clusters available for strong lensing, but the requirement that arcs should be large imposes a lower limit on the cluster masses. We see the combined effect in Figs. 5 and 6. If we set the cutoff radius to $\theta_{\min }=10^{\prime \prime}$, we select for highermass clusters in the first place, whose mass function is steeper than for lower-mass clusters. Thus, the effect of lowering the lower mass limit by increasing the halo concentrations is more pronounced if the cutoff radius is chosen higher.

Increasing the ellipticity of the lensing potential increases its gravitational tidal field, or shear. The increase in halo concentration caused by the earlier halo formation in dark energy models with $w>-1$ is then relatively less important for arc 
formation, which explains why the arc optical depth caused by cluster populations with lower $e$ changes more strongly with $w$ than for clusters with higher $e$. Fitting the elliptical NFW model to numerically simulated clusters yields values for $e$ closer to 0.3 .

\section{Summary and conclusions}

We investigated how the probability for the formation of large gravitational arcs in galaxy clusters is expected to change as the underlying cosmological model is modified. The main reason for this investigation was our earlier finding that halo concentrations in a simple class of spatially flat, dark-energy dominated cosmological models are expected to depend sensitively on the equation-of-state parameter $w$ (Bartelmann et al. 2002). Strong lensing in general, and the formation of large arcs in particular, is a highly nonlinear effect which depends sensitively on the matter concentration in the lens cores. It could therefore reasonably be expected that arc probabilities would change significantly with $w$.

This is of cosmological relevance since earlier numerical simulations (Bartelmann et al. 1998) showed that clusters in cosmological-constant models fall short by about an order of magnitude of reproducing the abundance of observed large gravitational arcs. While virtually all recent cosmological experiments favour a spatially flat, low-density universe, arc statistics apparently strongly prefers a low-density, open model over a low-density model with cosmological constant. This numerical result was questioned on the basis of simple analytic models which failed to reproduce the strong dependence of arc statistics on $\Omega_{\Lambda}$ found in the simulations (Cooray 1999; Kaufmann \& Straumann 2000). Our first goal was thus to investigate whether the numerical results could be supported by a more detailed analytic model.

Earlier studies also showed that analytic models for arc cross sections are notoriously problematic and inadequate for an accurate absolute quantification of arc probabilities. However, since it was our main interest in this paper to quantify changes in the arc optical depth relative to cosmologicalconstant models, we applied reasonably flexible analytic lens models which we demonstrate to reproduce the relevant features of fully numerical results (e.g. Bartelmann et al. 1998; Meneghetti et al. 2003), as described below.

We have chosen to use lenses with NFW density profile whose lensing potential was elliptically distorted. This model has several advantages. First, it naturally incorporates the dependence of central halo concentration on cosmology, through the halo formation time. Second, it agrees with density profiles consistently found in numerical simulations. Third, the elliptical distortion strongly decreases the mismatch between analytic and fully numerical lens models (Meneghetti et al. 2003). Although elliptical lensing potentials can lead to locally negative surface mass densities for sufficiently large ellipticities, this is a mild effect which happens only well outside the core for our model, thus it should be irrelevant for our purposes. We further adopted a simple estimate for the arc cross section and verified that this estimate scaled with cluster mass and dark energy parameter $w$ in the same way as fully numerically determined arc cross sections do. In particular, our simple analytic description for the arc cross section well reproduces the approximately quadratic scaling with cluster mass obtained fully numerically from the same elliptically distorted NFW cluster mass models. Although we would not trust this model for any accurate quantitative prediction of arc probabilities, we are confident that it can be used for the relative statements intended here.

Our main findings are as follows:

- Our simple lens model is indeed capable of reproducing the order-of-magnitude difference between $\Lambda \mathrm{CDM}$ and open CDM models found in the earlier numerical study. This shows that the change in halo concentration between the two models can explain the sensitivity of arc statistics for the cosmological constant. An additional effect into the same direction is contributed by the steep mass function of galaxy clusters. An increase in halo concentration lowers the minimum mass required for significant strong lensing, and this makes many more halos available for arc formation.

- Although increasing the dark energy parameter $w$ has a noticeable effect on the optical depth for arc formation, it cannot increase the arc optical depth to a level compatible with that found in open CDM models. This result arises due to a combination of three main effects: first, individual halos of fixed mass get more concentrated in dark energy than in cosmological constant models; second, lower mass halos than before become able to form large arcs; and third, the requirement that arcs be large imposes a lower limit on cluster masses. Our analytic model thus suggests that arc statistics cannot be reconciled with low-density, spatially flat cosmological models which are now dominated by dark energy, i.e. the discrepancy between arc statistics and the cosmological model favoured by most, if not all, recent cosmological experiments is not expected to disappear if the dark energy is not a cosmological constant, but has an equation of state $p=w \rho c^{2}$ with $w>-1$.

Being based on several simple analytic estimates, this study can only provide a tentative answer. Detailed numerical simulations will be necessary for reliable absolute quantifications of the arc optical depth expected in cosmological models with dark energy instead of a cosmological constant; similarly, it is necessary to quantify the dependence of the effect we find here on the specific dark energy model considered, such as a quintessence scalar field.

According to our preliminary analytic results presented here, it appears that the solution to the arc statistics problem as described by Bartelmann et al. (1998) is probably not to be found in the cosmological model alone, but more in the details of cluster structure and the history of cluster evolution. The recent suggestion by Wambsganss et al. (2003) that it could simply be removed by considering higher-redshift sources is certainly not a viable solution because the problem arose from comparing simulations to a well-defined sample of highly X-ray luminous clusters which were observed for arcs down to a well-defined photometric limit (Le Fèvre et al. 1994; Luppino et al. 1999). In combination with other recent 
results on the strong-lensing properties of dark-matter halos (e.g. Oguri et al. 2001; Oguri 2002; Oguri et al. 2003) and the puzzling discoveries of very high-redshift arcs in high-redshift clusters (Zaritsky \& Gonzalez 2003; Gladders et al. 2003), this seems to imply that arc statistics are teaching us that the properties of individual clusters and their evolution over time is still insufficiently understood.

Acknowledgements. We wish to thank Peter Schneider, Achim Weiss and Simon White for useful discussions and comments. This work has been partially supported by Italian MIUR (Grant 2001, prot. 2001028932, "Clusters and groups of galaxies: the interplay of dark and baryonic matter"), CNR and ASI.

\section{References}

Abroe, M., Balbi, A., Borrill, J., et al. 2002, MNRAS, 334, 11 Bardeen, J. M., Bond, J. R., Kaiser, N., \& Szalay, A. S. 1986, ApJ, 304, 15

Bartelmann, M. 1996, A\&A, 313, 697

Bartelmann, M., Ehlers, J., \& Schneider, P. 1993, A\&A, 280, 351

Bartelmann, M., Huss, A., Colberg, J., Jenkins, A., \& Pearce, F. 1998, A\&A, 330, 1

Bartelmann, M., Perrotta, F., \& Baccigalupi, C. 2002, A\&A, 396, 21

Bartelmann, M., Steinmetz, M., \& Weiss, A. 1995, A\&A, 297, 1

Bartelmann, M., \& Weiss, A. 1994, A\&A, 287, 1

Bullock, J., Kolatt, T., Sigad, Y., et al. 2001, MNRAS, 321, 559

Carroll, S. 2001, Living Rev., 4, 1

Chiba, M., \& Yoshii, Y. 1999, ApJ, 510, 42

Cooray, A. 1999, ApJ, 524, 504

Douspis, M., Riazuelo, A., Zolnierowski, Y., \& Blanchard, A. 2003, A\&A, 405, 409

Efstathiou, G., Moody, S., Peacock, J., et al. 2002, MNRAS, 330, 29

Eke, V., Navarro, J., \& Steinmetz, M. 2001, ApJ, 554, 114

Falco, E., Kochanek, C., \& Muñoz, J. 1998, ApJ, 494, 47

Flores, R., Maller, A., \& Primack, J. 2000, ApJ, 535, 555

Flores, R., \& Primack, J. 1994, ApJ, 427, L1

Gioia, I., \& Luppino, G. 1994, ApJS, 94, 583

Gladders, M., Hoekstra, H., Yee, H., Hall, P., \& Barrientos, L. 2003, ApJ, submitted [astro-ph/0303341]

Golse, G., \& Kneib, J.-P. 2002, A\&A, 390, 821

Halverson, N., Leitch, E., Pryke, C., et al. 2002, ApJ, 568, 38

Helbig, P. 1999, A\&A, 350, 1

Kassiola, A., \& Kovner, I. 1993, ApJ, 417, 450

Kaufmann, R., \& Straumann, N. 2000, Ann. Phys., 9, 384

Keeton, C. 2002, ApJ, 575, L1
Kochanek, C. 1996, ApJ, 466, 638

Kochanek, C., \& White, M. 2001, ApJ, 559, 531

Lacey, C., \& Cole, S. 1993, MNRAS, 262, 627

Lacey, C., \& Cole, S. 1994, MNRAS, 271, 676

Le Fèvre, O., Hammer, F., Angonin, M., Gioia, I., \& Luppino, G. 1994, ApJ, 422, L5

Lee, A., Ade, P., Balbi, A., et al. 2001, ApJ, 561, L1

Lehár, J., Falco, E., Kochanek, C., et al. 2000, 536, 584

Luppino, G., Gioia, I., Hammer, F., Le Fèvre, O., \& Annis, J. 1999, A\&AS, 136, 117

Mainini, R., Macciò, A., \& Bonometto, S. 2003, New Astron., 8, 173

Melchiorri, A., Mersini, L., Odman, C., \& Trodden, M. 2003, preprint [astro-ph/0211522]

Meneghetti, M., Bartelmann, M., \& Moscardini, L. 2003, MNRAS, 340,105

Meneghetti, M., Bolzonella, M., Bartelmann, M., Moscardini, L., \& Tormen, G. 2000, MNRAS, 314, 338

Meneghetti, M., Yoshida, N., Bartelmann, M., et al. 2001, MNRAS, 325,435

Navarro, J., Frenk, C., \& White, S. 1996, ApJ, 462, 563

Navarro, J., Frenk, C., \& White, S. 1997, ApJ, 490, 493

Netterfield, C., Ade, P., Bock, J., et al. 2002, ApJ, 571, 604

Oguri, M. 2002, ApJ, 573, 51

Oguri, M., Lee, J., \& Suto, Y. 2003, ApJ, submitted [astro-ph/0306102]

Oguri, M., Taruya, A., \& Suto, Y. 2001, ApJ, 559, 572

Peebles, P., \& Ratra, B. 2002, Rev. Mod. Phys., 75, 599

Perlmutter, S., Aldering, G., Goldhaber, G., et al. 1999, ApJ, 517, 565

Porciani, C., \& Madau, P. 2000, 532, 679

Press, W., \& Schechter, P. 1974, ApJ, 187, 425

Quast, R., \& Helbig, P. 1999, A\&A, 344, 721

Reiprich, T., \& Böhringer, H. 2002, ApJ, 567, 716

Richstone, D., Loeb, A., \& Turner, E. L. 1992, ApJ, 393, 477

Riess, A. G., Filippenko, A. V., Challis, P., et al. 1998, AJ, 116, 1009

Sheth, R., \& Tormen, G. 1999, MNRAS, 308, 119

Smoot, G. 1999, in AIP Conf. Proc., 476, 3K Cosmology, ed. L. Maiani, F. Melchiorri, \& N. Vittorio

Spergel, D., Verde, L., Peiris, H., et al. 2003, ApJ, submitted [astro-ph/0302209]

Wambsganss, J., Bode, P., \& Ostriker, J. 2003, ApJ, submitted [astro-ph/0306088]

Wang, L., \& Steinhardt, P. 1998, ApJ, 508, 483

Wang, X., Tegmark, M., \& Zaldarriaga, M. 2002, Phys. Rev. D, 65, 123001

Wu, X.-P., \& Mao, S. 1996, ApJ, 463, 404

Zaritsky, D., \& Gonzalez, A. 2003, ApJ, 584, 691 